\title{
Bioinformatics analysis to identify the differentially expressed genes of glaucoma
}

\author{
XIANG YAN $^{1}$, FEI YUAN ${ }^{2}$, XIUPING CHEN ${ }^{2}$ and CHUNQIONG DONG $^{2}$ \\ ${ }^{1}$ Department of Ophthalmology, Pudong District Gongli Hospital, Shanghai 200135; \\ ${ }^{2}$ Department of Ophthalmology, Zhongshan Hospital, Fudan University, Shanghai 200032, P.R. China
}

Received August 29, 2014; Accepted May 6, 2015

DOI: $10.3892 / \mathrm{mmr} .2015 .4030$

\begin{abstract}
The aim of the present study was to screen the differentially expressed genes (DEGs) associated with glaucoma and investigate the changing patterns of the expression of these genes. The GSE2378 gene microarray data of glaucoma was downloaded from the Gene Expression Omnibus database, which included seven normal samples and eight glaucoma astrocyte samples. Taking into account the corresponding associations between the probe ID and gene symbols, the DEGs were identified prior to and subsequent to the summation of probe level values using the Limma package in R language, followed by Gene Ontology (GO) and pathway enrichment analyses. Interaction networks of the DEGs were constructed using the Biomolecular Interaction Network Database, and cluster analysis of the genes in the networks was performed using ClusterONE. Subsequent to the summation of probe value, a total of 223 genes were identified as DEGs between the normal and glaucoma samples, including 74 downregulated and 149 upregulated genes. In addition, the DEGs were found to be associated with several functions, including response to wounding, extracellular region part and calcium ion binding. The most significantly enriched pathways were complement and coagulation cascades, arrhythmogenic right ventricular cardiomyopathy and extracellular matrix (ECM)-receptor interaction. Furthermore, interaction networks were constructed of the DEGs prior to and subsequent to the summation of probe values, and $H N F 4 A$ and $C E B P D$ were identified as hub genes. Additionally, 37 and 31 GO terms were identified to be enriched in the two DEGs of the networks prior to and subsequent to summation, respectively. The results indicated the identified genes associated with ECM as important, and the CEBPD gene was considered to be a critical gene in glaucoma. The findings of the present
\end{abstract}

Correspondence to: Dr Fei Yuan, Department of Ophthalmology, Zhongshan Hospital, Fudan University, 180 Fenglin Road, Shanghai 200032, P.R. China

E-mail: yuanfei20@yahoo.com

Key words: glaucoma, astrocytes, extracellular matrix, gene chip, differently expressed genes study offer a potential reference value in further investigations of glaucoma at the gene level.

\section{Introduction}

Glaucoma is an ocular disorder, characterized by intraocular pressure-associated optic neuropathy, with open angle and closed angle glaucoma being the two predominant types. At present, glaucoma is the second leading cause of vision loss worldwide (1). The number of individuals with open angle glaucoma worldwide in 2000 was 44,700,000, and the number is projected to increase to $79,600,000$ worldwide (1). In 2013, the population of patients aged between 40 and 80 years with glaucoma worldwide was estimated to be $64,300,000$ (2).

The chronic increase in intraocular pressure, which results in eye pain, is considered a key risk factor for glaucoma (3). Dysfunction of the corneal endothelium results in bullous keratopathy, characterized by progressive optic nerve fiber loss and retinal ganglion cell death (4). In glaucoma, optic nerve fiber degeneration initially occurs at the lamina cribrosa (5), which is formed by extracellular matrix (ECM) and quiescent astrocytes (6,7), and functions as a fibroelastic structure, providing mechanical and biological support for optic nerve axons. Chronic elevated intraocular pressure results in ECM remodeling and activation of quiescent astrocytes (8). In turn, the reactive astrocytes express new ECM proteins, a number of which are considered to alter its composition or be neurotoxic to the retinal ganglion cells.

There is a genetic basis underlying a substantial fraction of glaucoma. It has been reported that $\sim 5 \%$ of primary open angle glaucoma cases are currently attributed to single-gene or Mendelian forms of glaucoma (9). The vascular endothelial growth factor (VEGF) family and collagen gene family have been associated with glaucoma risk (10). It has been reported that the VEGF family consists of positive regulators of angiogenesis in the retina $(11,12)$. In addition, VEGF has been demonstrated to be a key inducer of corneal neovascularization (13-15), which may contribute to the further understanding and treatment of glaucoma. Previous studies have implicated the involvement of collagen genes in the regulation of central corneal thickness, which is a risk factor of glaucoma and, thus, possibly associated with the pathogenesis of glaucoma (16). Variations in collagen genes, which lead to inter-individual differences in scleral and lamina cribrosa properties, have 
been previously reported to result in different susceptibilities of individuals to elevated intraocular pressure (17). Therefore, it has been suggested that collagen mutations may cause glaucoma (18). Each of these investigations concerning glaucoma genetics have provided novel insights into gene therapy, which appears to be a promising approach in the treatment of glaucoma (19).

Genome-wide analyses of glaucoma have been performed. Bettahi et al (20) selected the differentially expressed genes (DEGs) in healing corneal epithelial cells of normal, vs. diabetic corneas. Pieragostino et al (21) examined differential protein expression in the tears of patients with pseudoexfoliative and primary open angle glaucoma. Microarray data in leukocytes of patients with primary open angle glaucoma has also been analyzed to examine variations at a genetic level (22). The GSE2378 gene expression profile in the Gene Expression Omnibus (GEO) database is comprised of seven and eight astrocyte samples from donors with and without glaucoma, respectively, and has been previously downloaded to screen DEGs and cluster-associated functions (23-25). However, the interaction among DEGs, particularly the functional modules in the interaction network, remain to be elucidated.

In the present study, the GSE2378 gene expression array was used and, to eliminate the effects of mismatching between large quantities of probe IDs and gene symbols, the data were divided into two groups: Prior to and following the summation of probe values. The DEGs were screened, followed by Gene Ontology (GO) and pathway enrichment analysis and, to examine the potential mechanism of glaucoma, interactions between the DEGs were investigated and visualized and significant functional modules in the network were assessed.

\section{Materials and methods}

Derivation of genetic data. The GSE2378 gene expression profiles of optic nerve astrocytes $(26,27)$ were downloaded from the public functional genomics data repository GEO database (http://www.ncbi.nlm.nih.gov/geo/) (28). In total, 15 specimens, including seven normal samples and eight glaucoma specimens, were available, based on the Human Genome U95 version 2 array from Affymetrix, Inc. (Santa Clara, CA, USA).

Normalization of data. The original GSE2378 data in the CEL files were converted into expression measures using the affy package in R language (29) (http://www.bioconductor. org/packages/3.0/bioc/), and background correction and quartile data normalization were performed using the robust multiarray average algorithm with default parameters in the $\mathrm{R}$ affy package $(30,31)$.

Selection of DEGs. The Limma package in R (32) (http://www.bioconductor.org/packages/release/bioc/html/limma.html) was used to identify the DEGs at the probe level between the glaucoma samples and normal samples. $\mathrm{P}<0.01$ and $\log$ fold change (FC) $>0.5$ were used as the cut-off criteria. The DEGs were determined pre- and post-summation of the probe value. In the treatment of post-summation of probe value, when multiple probe sets corresponded to the same gene, the expression values of the probes were added as the final value of gene expression for the differential expression screening.

Function and pathway enrichment of the DEGs. Functional enrichment analysis was conducted for DEGs, to identify changes in biological function or characteristics by calculating the whole significance of the gene expression (33). Gene-annotation enrichment analysis is a high-throughput strategy, which reduces the dimension of the data analysis and increases the likelihood of identifying the most relevant biological processes, making it a common approach in functional investigations of large-scale genomic or microarray data (34). Although a number of high-throughput enrichment tools can provide gene function enrichment analysis, the most widely used is Database for Annotation, Visualization and Integration Discovery (DAVID) (35) (http://david.abcc. Ncifcrf.gov/). In the present study, DAVID was applied to the enriched GO categories, based on a hypergeometric distribution with a count (gene number enriched in a specific GO term) $>5$ and the false discovery rate (FDR) $<0.01$. In addition, the over-represented Kyoto Encyclopedia of Genes and Genomes (KEGG) categories in the pathways (36) were identified.

Protein-protein interaction network and functional module analysis. The Biomolecular Interaction Network Database (BIND; http://bind.ca) (37) archives biomolecular interaction, complex and pathway information. Continued input from users has further improved the BIND data specification, which includes the ability to store detailed information about genetic interactions. Based on the available gene information of the DEGs in the above dataset, the interaction networks were analyzed using Cytoscape (http://www.cytoscape. org/) with a confidence threshold of 0.7 . In addition, cluster analysis of genes in protein-protein interaction networks was performed to identify modules with the highest confidence levels using ClusterONE (http://www.paccanarolab. org.sci-hub.org/clusterone/) in the Cytoscape software. Subsequently, GO enrichment analysis of the clustered genes in the selected module was performed, using DAVID with parameters of count $>5$ and the $\mathrm{FDR}<0.01$.

\section{Results}

Identification of DEGs. Based on the Limma package in $\mathrm{R}$ language, using $\mathrm{P}<0.01$ and $\mid \log \mathrm{FCl}>0.5$ cut-offs, a total of 234 probes were identified to be differentially expressed in the glaucoma samples compared with the normal control samples, which included 79 downregulated probes, corresponding to 67 genes; and 155 upregulated probes, corresponding to 142 genes. A total of four probes matching the MYH11 gene were significantly downregulated. Subsequent to statistical analysis, 2,000 genes were identified to match multiple probes. Accordingly, the expression profiles of the probes were added for the same gene to perform the differential analysis between the normal and glaucoma groups at the gene expression level, rather than at the probe level only. In total, 223 DEGs were identified post-summation of the probe value, including 74 downregulated and 149 upregulated genes. Compared with the results pre-summation, there were 
Table I. Differentially expressed genes pre- and post-summation.

\begin{tabular}{|c|c|c|c|c|c|c|}
\hline \multirow[b]{2}{*}{ Probe ID } & \multicolumn{3}{|c|}{ Pre-summation } & \multicolumn{3}{|c|}{ Post-summation } \\
\hline & Gene symbol & $\log \mathrm{FC}$ & P-value & Gene symbol & $\operatorname{logFC}$ & P-value \\
\hline 32582_at & MYH11 & -3.00 & $6.37 \mathrm{E}-04$ & MYH11 & -8.96 & $1.44 \mathrm{E}-03$ \\
\hline 34235_at & GPR116 & -2.36 & 0.007138566 & ITGA6 & -3.06 & $5.35 \mathrm{E}-04$ \\
\hline 37407_s_at & MYH11 & -2.29 & 0.002362727 & STAT1 & -2.54 & $2.13 \mathrm{E}-03$ \\
\hline 767_at & MYH11 & -1.97 & 0.003229233 & GPR116 & -2.36 & $7.83 \mathrm{E}-03$ \\
\hline 40488_at & DMD & -1.49 & $9.75 \mathrm{E}-04$ & RBPMS & -2.14 & $4.54 \mathrm{E}-03$ \\
\hline 39710_at & NREP & -1.48 & 0.003015513 & CSPG4 & -1.90 & $1.30 \mathrm{E}-03$ \\
\hline 37279_at & GEM & -1.40 & $1.35 \mathrm{E}-03$ & SLC1A1 & -1.85 & $8.53 \mathrm{E}-03$ \\
\hline 38004_at & CSPG4 & -1.38 & 0.000514187 & TEK & -1.82 & $2.11 \mathrm{E}-03$ \\
\hline 40899_at & KRT19 & -1.27 & 0.007598562 & ITGA3 & -1.63 & $2.60 \mathrm{E}-03$ \\
\hline 774_g_at & MYH11 & -1.23 & 0.005252688 & PDLIM5 & -1.57 & 7.72E-03 \\
\hline 41215_s_at & 41215_s_at & 2.05 & 0.007785332 & SEPP1 & 2.43 & $9.40 \mathrm{E}-05$ \\
\hline 36686_at & ALDH1A3 & 2.09 & $5.06 \mathrm{E}-03$ & ADH1B & 2.48 & $1.51 \mathrm{E}-06$ \\
\hline 38379_at & GPNMB & 2.18 & 0.002595973 & CLU & 2.49 & $1.24 \mathrm{E}-03$ \\
\hline 1380_at & FGF7 & 2.25 & 4.40E-03 & PDE1A & 2.77 & $2.28 \mathrm{E}-03$ \\
\hline 34363_at & SEPP1 & 2.43 & 7.77E-05 & AKR1C3 & 2.88 & $8.90 \mathrm{E}-05$ \\
\hline 35730_at & ADH1B & 2.49 & $1.14 \mathrm{E}-06$ & 32805_at & 3.25 & $5.81 \mathrm{E}-04$ \\
\hline 36780_at & CLU & 2.49 & 0.00108886 & ID1 & 3.45 & $1.26 \mathrm{E}-03$ \\
\hline 36311_at & PDE1A & 2.78 & 0.00202481 & CTSK & 3.56 & $6.69 \mathrm{E}-05$ \\
\hline 37399_at & AKR1C3 & 2.89 & $7.35 \mathrm{E}-05$ & PTGDS & 4.63 & $1.37 \mathrm{E}-04$ \\
\hline 32805_at & 32805_at & 3.25 & $5.00 \mathrm{E}-04$ & FGF7 & 6.20 & $2.18 \mathrm{E}-03$ \\
\hline
\end{tabular}

Top 10 differentially expressed genes were determined based on a logFC values $>0.5$. FC, fold change

189 DEGs in common, with the most significant gene listed in Table I.

The MYH1, CSPG41 and GPR116 genes were identified to be the most significantly downregulated DEGs prior and subsequent to probe value summation. Similarly, among the upregulated genes, FGF 7, ADH1B, CLU, ARRIC3, SEPP1 and $P D E 1 A$ were in the top 10 significant DEGs. Scatter diagrams of pre- and post-summation data demonstrated that no significant difference existed in the number of DEGs (Fig. 1A). Excluding the repeated genes, the common DEGs pre- and post-summation of probe value were revealed using Venn analysis (Fig. 1B). A total of 128 common upregulated genes and 61 downregulated genes were identified. No genes contradicted each other in the four categories.

Enrichment analysis of the DEGs. To determine the functions of DEGs in glaucoma, the 189 common DEGs were mapped to the GO database. GO terms in biological process (BP), including response to wounding, regulation of cell proliferation and vasculature development; terms in cellular component (CC), including extracellular region part, extracellular region and cytoplasmic membrane-bounded vesicle lumen; and terms in molecular function (MF), including calcium ion binding, carbohydrate binding and calmodulin binding, were enriched (Table II).

In order to further investigate changes to the biological pathways in glaucoma cells, the significant pathways associated with the DEGs were identified. The five pathways identified with significant P-values are listed in Table III. The most significant enrichment pathways were complement and coagulation cascades, arrhythmogenic right ventricular cardiomyopathy and ECM-receptor interaction.

Interactive network analysis. The DEGs were mapped to the BIND database and significant interactions were screened with a confidence coefficient $>0.7$. By integrating these associations, interaction networks of the DEGs were constructed. In the networks of DEGs prior to summation (Fig. 2Aa), $H N F 4 A$ was connected with multiple modules. The protein in the network serves as a node, and the degree of a node denotes the number of proteins interacting with the specific node, which is indicated by the lines between them. The 'hub nodes' were defined as the nodes which had high degrees within the network. The IGF1R, RUNXIT1 and STAT1 DEGs were identified as hub nodes. Following cluster analysis using ClusterONE, a module containing FOS and CEBPD DEGs, and non-DEGs belonging to the $H N F 4 A$ and $C E B P$ families, were obtained (Fig. 2Ab). The module contained a total of 18 nodes, with a module density of 0.542 , quality of 0.874 and P-value of 2.222E-7.

The networks of the post-summation DEGs are shown in Fig. 2Ba and b. HNF4A was connected with multiple modules, and the HDACl and EGFR DEGs were identified as the hub nodes. Following cluster analysis, a module of 12 nodes, with a density of 0.758 , quality of 0.847 and P-value of $1.765 \mathrm{E}-5$ was obtained, including one DEG (CEBPD) (Fig. 2Bb). 
Table II. Top five significantly enriched GO terms of the differentially expressed genes.

\begin{tabular}{llrrr}
\hline Category & \multicolumn{1}{c}{ Term } & Count & P-value & Fold enrichment \\
\hline BP & GO:0009611 response to wounding & 27 & $5.08 \mathrm{E}-09$ & 3.893572 \\
BP & GO:0042127 regulation of cell proliferation & 33 & $7.11 \mathrm{E}-09$ & 3.20479 \\
BP & GO:0001944 vasculature development & 16 & $9.78 \mathrm{E}-07$ & 4.871992 \\
BP & GO:0006954 inflammatory response & 18 & $1.16 \mathrm{E}-06$ & 4.233012 \\
BP & GO:0007167 enzyme linked receptor protein & 17 & $1.01 \mathrm{E}-05$ & 3.799121 \\
& signaling pathway & & & $1.20 \mathrm{E}-05$ \\
CC & GO:0044421 extracellular region part & 31 & $2.19 \mathrm{E}-06$ & 2.579701 \\
CC & GO:0005576 extracellular region & 44 & $1.71 \mathrm{E}-04$ & 1.748781 \\
CC & GO:0060205 cytoplasmic membrane-bounded & 6 & $2.05 \mathrm{E}-04$ & 10.89375 \\
& vesicle lumen & & & $2.84 \mathrm{E}-03$ \\
CC & GO:0031983 vesicle lumen & 6 & $2.54 \mathrm{E}-04$ & $2.22 \mathrm{E}-01$ \\
CC & GO:0005615 extracellular space & 21 & $3.24 \mathrm{E}-04$ & $2.66 \mathrm{E}-01$ \\
MF & GO:0005509 calcium ion binding & 24 & $1.58 \mathrm{E}-03$ & 2.449106 \\
MF & GO:0030246 carbohydrate binding & 13 & $2.09 \mathrm{E}-03$ & 2.018187 \\
MF & GO:0005516 calmodulin binding & 8 & $2.21 \mathrm{E}-03$ & 2.837957 \\
MF & GO:0005539 glycosaminoglycan binding & 8 & $2.21 \mathrm{E}-03$ & 4.415986 \\
MF & GO:0003779 actin binding & 12 & $3.29 \mathrm{E}-03$ & 4.415986 \\
& & & 2.844654
\end{tabular}

BP, biological process; CC, cellular component; MF, molecular function; GO, Gene Ontology; FDR, false discovery rate.

A

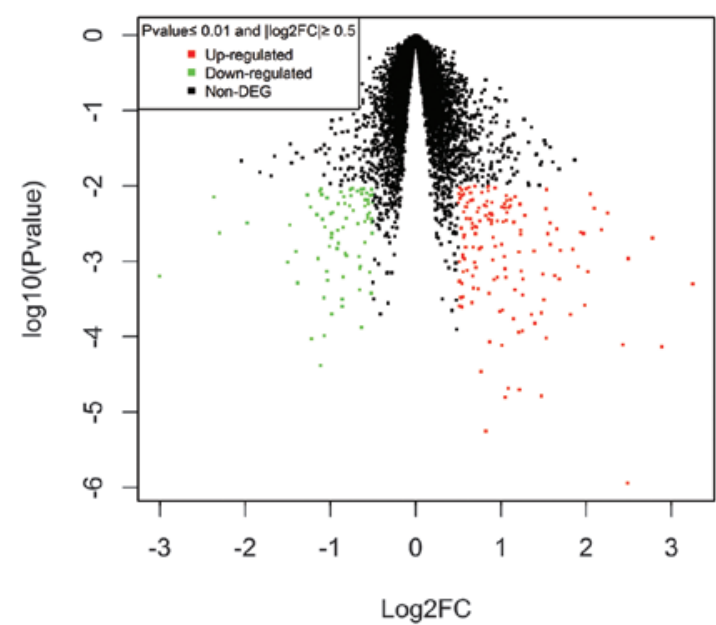

$\mathbf{b}$

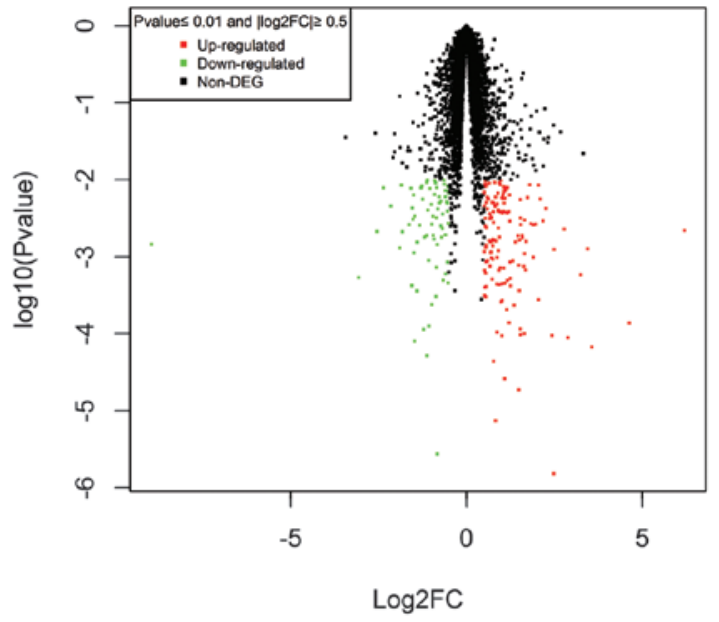

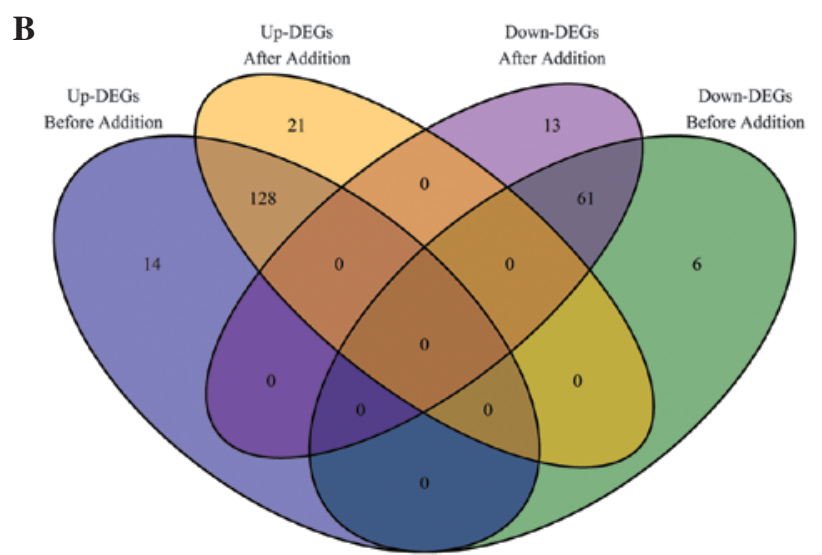

Figure 1. Gene expression analysis pre- and post-summation. (A) Scatter diagram of the gene expressing profiles (Aa) pre- and (Ab) post-summation. The $\log 2 \mathrm{FC}$ value is on the $\mathrm{x}$-axis and $\log 10$ (P-value) is on the $\mathrm{y}$-axis. (B) Comparison between the number of genes pre- and post-summation. DEGs, differentially expressed genes; FC, fold-change; Up-DEGs, upregulated DEGs; Down-DEGs, downregulated DEGs. 
Table III. Top five significantly enriched KEGG pathways of differentially expressed genes.

\begin{tabular}{lrrr}
\hline KEGG term & Count & P-value & Fold enrichment \\
\hline hsa04610:Complement and coagulation cascades & 10 & $2.96 \mathrm{E}-06$ & 8.010397 \\
hsa05412:Arrhythmogenic right ventricular cardiomyopathy & 8 & $3.73 \mathrm{E}-04$ & 5.818078 \\
hsa04512:Extracellular matrix-receptor interaction & 8 & $6.89 \mathrm{E}-04$ & 5.263975 \\
hsa05200:Pathways in cancer & 15 & $1.86 \mathrm{E}-03$ & 2.527671 \\
hsa04510:Focal adhesion & 11 & $2.92 \mathrm{E}-03$ & 3.024822 \\
\hline
\end{tabular}

KEGG, Kyoto Encyclopedia of Genes and Genomes; FDR, false discovery rate.
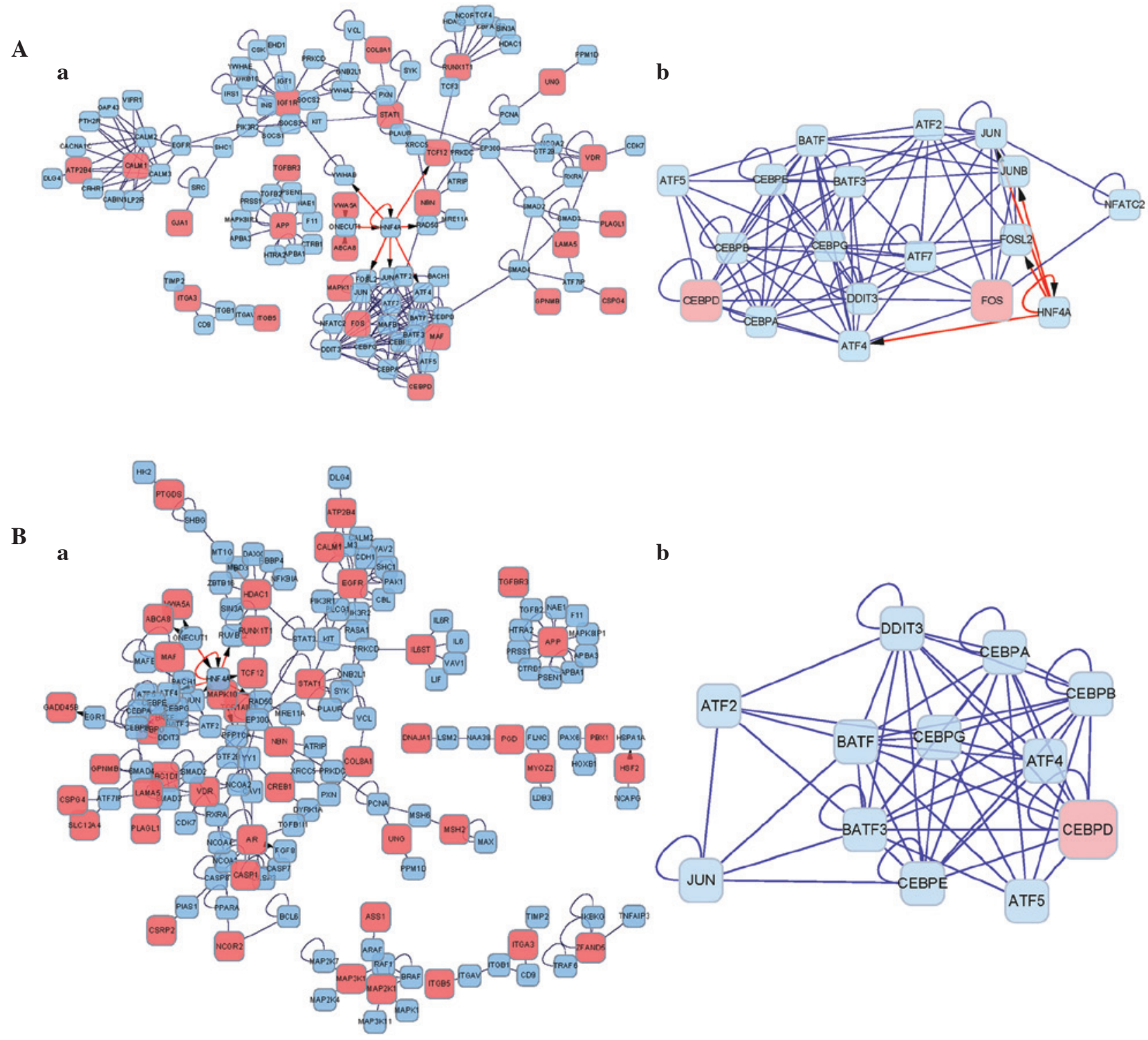

b

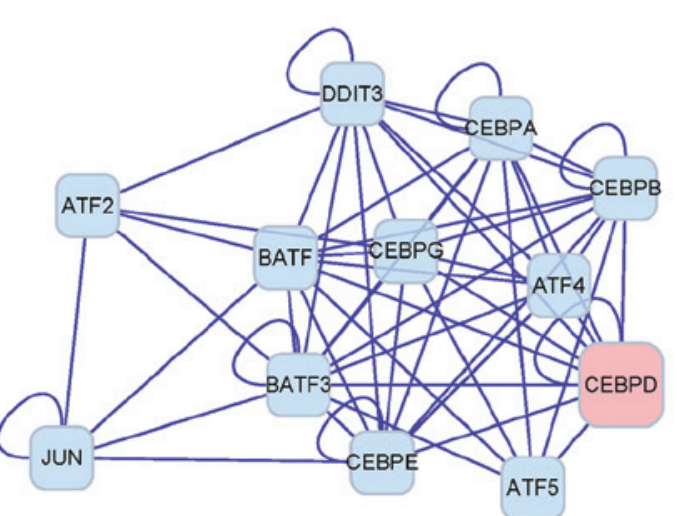

Figure 2. Interaction networks of the DEGs. (A) Pre-summation network; (Aa) whole network; (Ab) most significant subnetwork. (B) post-summation network; (Ba) whole network; (Bb) most significant subnetwork. Pink nodes represent DEGs; blue nodes represent their interaction genes. Red arrows indicate the interaction between protein and DNA, blue lines indicate interaction between proteins. DEGs, differentially expressed genes.

Functional annotation analysis of modules. Functional annotation analysis of the modules available in ClusterONE was performed. A total of 37 and $31 \mathrm{GO}$ terms were enriched in the two modules of the pre- and post-summation networks, respectively. The top three $\mathrm{BP}, \mathrm{CC}$ and MF enriched functions are listed in Table IV. The enriched genes were 
Table IV. Top three function enrichment terms of genes in the interaction network of differentially expressed genes.

\begin{tabular}{|c|c|c|c|c|c|}
\hline Category & Term & Count & P-value & $\begin{array}{l}\text { Fold- } \\
\text { enrichment }\end{array}$ & FDR \\
\hline \multicolumn{6}{|c|}{ Pre-summation } \\
\hline $\mathrm{BP}$ & $\begin{array}{l}\text { GO:0006355 regulation of transcription, } \\
\text { DNA-dependent }\end{array}$ & 18 & $9.29 \mathrm{E}-16$ & 7.630006 & $1.24 \mathrm{E}-12$ \\
\hline $\mathrm{BP}$ & $\begin{array}{l}\text { GO:0051252 regulation of RNA } \\
\text { metabolic process }\end{array}$ & 18 & $1.36 \mathrm{E}-15$ & 7.461666 & $1.87 \mathrm{E}-12$ \\
\hline $\mathrm{BP}$ & GO:0045449 regulation of transcription & 18 & $6.43 \mathrm{E}-13$ & 5.201077 & $8.98 \mathrm{E}-10$ \\
\hline $\mathrm{CC}$ & GO:0031981 nuclear lumen & 11 & $1.83 \mathrm{E}-08$ & 7.458992 & $1.46 \mathrm{E}-05$ \\
\hline $\mathrm{CC}$ & GO:0070013 intracellular organelle lumen & 11 & $1.35 \mathrm{E}-07$ & 6.079561 & $1.08 \mathrm{E}-04$ \\
\hline $\mathrm{CC}$ & GO:0043233 organelle lumen & 11 & $1.68 \mathrm{E}-07$ & 5.942604 & $1.35 \mathrm{E}-04$ \\
\hline MF & GO:0043565 sequence-specific DNA binding & 18 & $1.96 \mathrm{E}-23$ & 21.3888 & $1.89 \mathrm{E}-20$ \\
\hline MF & GO:0046983 protein dimerization activity & 17 & $1.12 \mathrm{E}-21$ & 22.6231 & $1.08 \mathrm{E}-18$ \\
\hline MF & GO:0003700 transcription factor activity & 18 & $6.75 \mathrm{E}-20$ & 13.3159 & $6.50 \mathrm{E}-17$ \\
\hline \multicolumn{6}{|c|}{ Post-summation } \\
\hline $\mathrm{BP}$ & $\begin{array}{l}\text { GO:0006355 regulation of transcription, } \\
\text { DNA-dependent }\end{array}$ & 12 & $1.91 \mathrm{E}-10$ & 7.630006 & $2.53 \mathrm{E}-07$ \\
\hline $\mathrm{BP}$ & $\begin{array}{l}\text { GO:0051252 regulation of RNA } \\
\text { metabolic process }\end{array}$ & 12 & $2.44 \mathrm{E}-10$ & 7.461666 & $3.23 \mathrm{E}-07$ \\
\hline $\mathrm{BP}$ & GO:0006350 transcription & 12 & $1.24 \mathrm{E}-09$ & 6.438839 & $1.64 \mathrm{E}-06$ \\
\hline $\mathrm{CC}$ & GO:0031981 nuclear lumen & 7 & $1.33 \mathrm{E}-05$ & 7.713276 & $1.06 \mathrm{E}-02$ \\
\hline $\mathrm{CC}$ & GO:0070013 intracellular organelle lumen & 7 & $4.45 \mathrm{E}-05$ & 6.286818 & $3.53 \mathrm{E}-02$ \\
\hline $\mathrm{CC}$ & GO:0043233 organelle lumen & 7 & $5.09 \mathrm{E}-05$ & 6.145192 & 4.03E-02 \\
\hline MF & GO:0046983 protein dimerization activity & 12 & $6.09 \mathrm{E}-16$ & 23.95387 & $5.00 \mathrm{E}-13$ \\
\hline MF & GO:0043565 sequence-specific DNA binding & 12 & $2.14 \mathrm{E}-15$ & 21.3888 & $1.88 \mathrm{E}-12$ \\
\hline MF & GO:0003700 transcription factor activity & 12 & $4.07 \mathrm{E}-13$ & 13.3159 & $3.63 \mathrm{E}-10$ \\
\hline
\end{tabular}

Top three BP, CC and MF terms, were determined based on the lowest P-values. BP, biological process; CC, cellular component; MF, molecular function; FDR, false discovery rate.

predominantly involved in the progress of gene transcription and expression.

\section{Discussion}

Among the selected DEGs, MYH11 was significantly downregulated pre- and post-summation. Notably, the four probes of MYH11 were all among the 10 most significantly downregulated genes. Accordingly, the different transcripts of MHY11 may be involved in the development of glaucoma. In addition, FGF7, ADHIB, CLU, ARR1C3, SEPPI and PDE1A were all significantly upregulated DEGs pre- and post-summation. Although a number of these have been reported to be involved in Alzheimer's disease or different types of cancer (38-43), there is little information regarding the systematic mechanism underlying the effect of these genes in glaucoma (44). Therefore, the functions of these genes require further investigation. The minimal difference between the pre- and post-summation DEGs, and the absence of contradiction between the upregulated and downregulated genes indicated the analysis used in the present study was reliable.

In the present study, GO functional annotation of the DEGs assisted in identifying associated genes involved in different biological progresses. In the BP term, functions associated with cell division and structure were enriched; in the $\mathrm{CC}$ term, functions associated with plasma lumen and vesicles were enriched; and in the MF term, the functions were predominantly involved in calcium signal transduction. These results reflected that the structures of the cell vesicles and microtubules were markedly altered in glaucoma, which was in accordance with the results of a previous study (4). DEGs were found to be enriched in the hsa04512: ECM-receptor interaction KEGG pathway, the genes of which have been reported to be closely associated with glaucoma (45).

In the interaction network analysis of the DEGs, $H N F 4 A$ was associated with multiple modules, indicating that this gene was important in regulating the expression of numerous genes and connecting various pathways. HNF4A has been reported to be associated with the pancreas and liver (46). A mutation in the $H N F-4 \mathrm{~A}$ gene has been reported to result in monogenic diabetes, of which glaucoma is a common complication (47). Therefore, further analysis of the association between $H N F 4 A$ and glaucoma is required. In addition, the roles of $C E B P D$, a member of $C E B P$ family, in the network confirmed the reliability of $\mathrm{GO}$ enrichment 
analysis, as GO:0042127: regulation of cell proliferation was significantly altered. It has been reported that the binding of the CCAAT enhancer to the $C E B P D$ transcription factor regulates the cell cycle (48) and its expression may inhibit the proliferation of tumor cells (49). In addition, cell proliferation, rather than astrocyte hypertrophy, characterizes early pressure-induced optic nerve head injury, leading to glaucoma (50). These findings suggested the possibility of identifying how the $C E B P D$ transcription factor assists in the inhibition of cell proliferation in glaucoma.

In conclusion, the present study identified DEGs using bioinformatics analysis and observed that CEBP family genes, in particular, $C E B P D$, may be important in the progression of glaucoma. Genes associated with the ECM were also suggested to be important. However, further experiments are required to confirm the results of the present study. Due to the increasing public availability of genomic data, similar approaches are likely to become more popular as a basis for future investigations.

\section{References}

1. Quigley HA and Broman AT: The number of people with glaucoma worldwide in 2010 and 2020. Br J Ophthalmol 90: 262-267, 2006

2. Tham YC, Li X, Wong TY, Quigley HA, Aung T and Cheng CY: Global prevalence of glaucoma and projections of glaucoma burden through 2040: A systematic review and meta-analysis. Ophthalmology 121: 2081-2090, 2014.

3. Caprioli J and Coleman AL: Intraocular pressure fluctuation a risk factor for visual field progression at low intraocular pressures in the advanced glaucoma intervention study. Opthalmology 115: 1123-1129, 2008.

4. Rudzinski MN, Chen L and Hernandez MR: Antiangiogenic characteristics of astrocytes from optic nerve heads with primary open-angle glaucoma. Arch Ophthalmol 126: 679-685, 2008.

5. Quigley HA: Neuronal death in glaucoma. Prog Retin Eye Res 18: 39-57, 1999

6. Hernandez MR: The optic nerve head in glaucoma: Role of astrocytes in tissue remodeling. Prog Retin Eye Res 19: 297-321, 2000

7. Varela HJ and Hernandez MR: Astrocyte responses in human optic nerve head with primary open-angle glaucoma. J Glaucoma 6: 303-313, 1997.

8. Liu B, Chen H, Johns TG and Neufeld AH: Epidermal growth factor receptor activation: An upstream signal for transition of quiescent astrocytes into reactive astrocytes after neural injury. J Neurosci 26: 7532-7540, 2006.

9. Fingert JH: Primary open-angle glaucoma genes. Eye (Lond) 25: 587-595, 2011.

10. Horsley MB and Kahook MY: Anti-VEGF therapy for glaucoma. Curr Opin Ophthalmol 21: 112-117, 2010.

11. Miller JW: Vascular endothelial growth factor and ocular neovascularization. Am J Pathol 151: 13-23, 1997.

12. Shima DT, Adamis AP, Ferrara N, et al: Hypoxic induction of endothelial cell growth factors in retinal cells: Identification and characterization of vascular endothelial growth factor (VEGF) as the mitogen. Mol Med 1: 182-193, 1995.

13. Ramazanoglu M, Lutz R, Ergun C, von Wilmowsky C, Nkenke E and Schlegel KA: The effect of combined delivery of recombinant human bone morphogenetic protein-2 and recombinant human vascular endothelial growth factor 165 from biomimetic calcium-phosphate-coated implants on osseointegration. Clin Oral Implants Res 22: 1433-1439, 2011.

14. Binder DR, Herring IP, Zimmerman KL, Pickett JP and Huckle WR: Expression of vascular endothelial growth factor receptor- 1 and -2 in normal and diseased canine eyes. Vet Ophthalmol 15: 223-230, 2012.

15. Chatterjee S, Wang Y, Duncan MK and Naik UP: Junctional adhesion molecule-A regulates vascular endothelial growth factor receptor-2 signaling-dependent mouse corneal wound healing. PloS One 8: e63674, 2013.
16. Vithana EN, Aung T, Khor CC, et al: Collagen-related genes influence the glaucoma risk factor, central corneal thickness. Hum Mol Genet 20: 649-658, 2011.

17. Desronvil T, Logan-Wyatt D, Abradou W, et al: Distribution of COL8A2 and COL8A1 gene variants in Caucasian primary open angle glaucoma patients with thin central corneal thickness. Mol Vis 16: 2185-2191, 2010.

18. Huang W, Fan Q, Wang W, Zhou M, Laties AM and Zhang X: Collagen: A potential factor involved in the pathogenesis of glaucoma. Med Sci Monit Basic Res 19: 237-240, 2013.

19. Liu X, Brandt CR, Rasmussen CA and Kaufman PL: Glaucoma gene therapy. Expert Rev Ophthalmol 2: 227-236, 2007.

20. Bettahi I, Sun H, Gao N, et al: Genome-wide transcriptional analysis of differentially expressed genes in diabetic, healing corneal epithelial cells: Hyperglycemia-suppressed TGF $\beta 3$ expression contributes to the delay of epithelial wound healing in diabetic corneas. Diabetes 63: 715-727, 2014.

21. Pieragostino D, Bucci S, Agnifili L, et al: Differential protein expression in tears of patients with primary open angle and pseudoexfoliative glaucoma. Mol Biosyst 8: 1017-1028, 2012.

22. Colak D, Morales J, Bosley TM, et al: Genome-wide expression profiling of patients with primary open angle glaucoma. Invest Ophthalmol Vis Sci 53: 5899-5904, 2012.

23. Liao Q, Sun X, Guo H and Li C: Exploring the potential mechanism and screening small molecule drugs for glaucoma by using bioinformatics approach. Eur Rev Med Pharmacol Sci 18: 132-140, 2014.

24. Shi WY, Liu KD, Xu SG, et al: Gene expression analysis of lung cancer. Eur Rev Med Pharmacol Sci 18: 217-228, 2014.

25. Liu T, Xie L, Ye J and He X: Family-based analysis identified $\mathrm{CD} 2$ as a susceptibility gene for primary open angle glaucoma in Chinese Han population. J Cell Mol Med 18: 600-609, 2014.

26. Hernandez MR, Agapova OA, Yang P, Salvador-Silva M, Ricard CS and Aoi S: Differential gene expression in astrocytes from human normal and glaucomatous optic nerve head analyzed by cDNA microarray. Glia 38: 45-64, 2002.

27. Kompass KS, Agapova OA, Li W, Kaufman PL, Rasmussen CA and Hernandez MR: Bioinformatic and statistical analysis of the optic nerve head in a primate model of ocular hypertension. BMC Neurosci 9: 93, 2008.

28. Edgar R, Domrachev M and Lash AE: Gene Expression Omnibus: NCBI gene expression and hybridization array data repository. Nucleic Acids Res 30: 207-210, 2002.

29. Chang-Zheng L, Xue-Hai M, Jin Z, et al: Posterior indirect-decompression technique versus posterior direct vision decompression for thoracolumbar burst fractures. Journal of Clinical Rehabilitative Tissue Engineering Research 15: 2011.

30. Troyanskaya O, Cantor M, Sherlock G, et al: Missing value estimation methods for DNA microarrays. Bioinformatics 17: 520-525, 2001.

31. Fujita A, Sato JR, Rodrigues Lde O, Ferreira CE and Sogayar MC: Evaluating different methods of microarray data normalization. BMC Bioinformatics 7: 469, 2006.

32. Smyth GK: Limma: Linear models for microarray data. In: Bioinformatics and Computational Biology Solutions Using $\mathrm{R}$ and Bioconductor. Gentleman R, Carey V, Huber W, Irizarry R and Dudoit S (eds). Springer, New York, pp397-420, 2005.

33. Zheng Q and Wang XJ: GOEAST: A web-based software toolkit for Gene Ontology enrichment analysis. Nucleic Acids Res 36 (Web Server Issue): W358-W363, 2008.

34. Huang da W, Sherman BT and Lempicki RA: Bioinformatics enrichment tools: Paths toward the comprehensive functional analysis of large gene lists. Nucleic Acids Res 37: 1-13, 2009.

35. Huang da W, Sherman BT and Lempicki RA: Systematic and integrative analysis of large gene lists using DAVID bioinformatics resources. Nat Protoc 4: 44-57, 2008.

36. Kanehisa M and Goto S: KEGG: Kyoto encyclopedia of genes and genomes. Nucleic Acids Res 28: 27-30, 2000.

37. Bader GD, Betel D and Hogue CW: BIND: The biomolecular interaction network database. Nucleic Acids Res 31: 248-250, 2003.

38. Lee CH, Lee JM, Wu DC, et al: Carcinogenetic impact of ADH1B and ALDH2 genes on squamous cell carcinoma risk of the esophagus with regard to the consumption of alcohol, tobacco and betel quid. Int J Cancer 122: 1347-1356, 2008.

39. Yasuhara T, Okamoto A, Kitagawa T, et al: FGF7-like gene is associated with pericentric inversion of chromosome 9 and FGF7 is involved in the development of ovarian cancer. Int J Oncol 26: 1209-1216, 2005. 
40. Yu JT, Li L, Zhu QX, et al: Implication of CLU gene polymorphisms in Chinese patients with Alzheimer's disease. Clin Chim Acta 411: 1516-1519, 2010.

41. Schlott T, Eiffert H, Bohne W, et al: Chlamydia trachomatis modulates expression of tumor suppressor gene caveolin-1 and oncogene $\mathrm{C}$-myc in the transformation zone of non-neoplastic cervical tissue. Gynecol Oncol 98: 409-419, 2005.

42. Méplan C, Hughes DJ, Pardini B, et al: Genetic variants in selenoprotein genes increase risk of colorectal cancer. Carcinogenesis 31: 1074-1079, 2010.

43. Abusnina A, Keravis T, Yougbaré I, Bronner C and Lugnier C: Anti-proliferative effect of curcumin on melanoma cells is mediated by PDE1A inhibition that regulates the epigenetic integrator UHRF1. Mol Nutr Food Res 55: 1677-1689, 2011.

44. Mansberger SL, MacKenzie PJ and Falardeau J: Optic disc cupping associated with neurosyphilis. J Glaucoma 22: 80-83, 2013.

45. Kirwan RP, Wordinger RJ, Clark AF and O'Brien CJ: Differential global and extra-cellular matrix focused gene expression patterns between normal and glaucomatous human lamina cribrosa cells. Mol Vis 15: 76-88, 2009.
46. Odom DT, Zizlsperger N, Gordon DB, et al: Control of pancreas and liver gene expression by HNF transcription factors. Science 303: 1378-1381, 2004.

47. Edghill EL and Hattersley AT: Genetic disorders of the pancreatic beta cell and diabetes (permanent neonatal diabetes and maturity-onset diabetes of the young). In: Pancreatic Beta Cell in Health and Disease. Seino S and Bell GI (eds) Springer Japan, Tokyo, Japan, pp399-430, 2008.

48. Ramji DP and Foka P: CCAAT/enhancer-binding proteins: Structure, function and regulation. Biochem J 365: 561-575, 2002.

49. Johnson PF: Molecular stop signs: Regulation of cell-cycle arrest by C/EBP transcription factors. J Cell Sci 118: 2545-2555, 2005.

50. Johnson EC, Doser TA, Cepurna WO, et al: Cell proliferation and interleukin-6-type cytokine signaling are implicated by gene expression responses in early optic nerve head injury in rat glaucoma. Invest Ophthalmol Vis Sci 52: 504-518, 2011. 